\title{
Identification and Characterization of in vitro Metabolites of Belinostat by Rat Liver Microsomes using Ultra Performance Liquid Chromatography Coupled with Tandem Mass Spectrometry
}

\author{
Parul Grover,"\#, Lovekesh Mehta ${ }^{2, *, \#, ~ T a n v e e r ~ N a v e d ², ~ S a n d e e p ~ K u m a r ~}{ }^{3}$, Gorav Monga ${ }^{4}$ \\ ${ }^{1}$ Department of Pharmaceutical Sciences, KIET School of Pharmacy, KIET Group of Institutions, Delhi-NCR, Ghaziabad, \\ Uttar Pradesh, INDIA. \\ ${ }^{2}$ Department of Pharmaceutical Sciences, Amity Institute of Pharmacy, Amity University, Noida, Uttar Pradesh, INDIA. \\ ${ }^{3}$ Department of Regulatory Affairs, National Institute of Pharmaceutical Education and Research, Hyderabad, Telangana, INDIA. \\ ${ }^{4}$ Department of DMPK and Bioanalytical, Dabur Research Foundation, Sahibabad, Ghaziabad, Uttar Pradesh, INDIA. \\ "Both Authors Contributed Equally
}

\begin{abstract}
Objectives: The objective of the present study is to study the in-vitro metabolites of FDA approved anticancer drug, belinostat which is a histone deacetylase inhibitor. Methods: The metabolites were formed by incubating the drug, belinostat with rat liver microsomes, at $37^{\circ} \mathrm{C}$ for $24 \mathrm{hr}$. The newly formed metabolites were identified and characterized with the help of ultra-high performance liquid chromatography which is interlinked with tandem quadrupole time-of-flight mass spectrometry. Results: Two Phase-I metabolites were formed which include a belinostat amide (reductive metabolite) and belinostat acid (deaminated belinostat). We then elucidated the structures of the formed metabolites based on LC-MS/MS data which included accurate masses, the fragmentation of ions and chromatographic retention times. Conclusion: This study describes the detailed in-vitro metabolite profiling of belinostat which will be further helpful to predict the in-vivo metabolism of belinostat in the human body. Enzyme kinetic parameters $\left(K_{\mathrm{m}}\right.$ and $\left.\mathrm{V}_{\max }\right)$ for both the metabolites were also established using different substrate concentrations. The study will be helpful in determining the safety and efficacy of the drug. This will further help in determining the elimination mechanism of belinostat which in turn will assist in predicting the effectiveness and toxicity of the drug.

Key words: Belinostat; Rat Liver Microsomes; Characterization; in-vitro Metabolites; Enzyme Kinetics; LC-MS/MS.
\end{abstract}

\section{INTRODUCTION}

In drug discovery process, one of the major challenges is to rapidly and accurately predict in vivo metabolism of drugs, their pharmacokinetics and toxicity. One of the convenient approaches, to predict possible metabolic repertoire, is in vitro drug metabolism and pharmacokinetic studies.

Several reactions take place in a living organism to sustain life. We define metabolism as a biotransformation process where various drugs are bio catalyzed into a different chemical form with the help of enzymes. The major sites of enzymatic metabolism in the body include the liver, kidney, intestine, lungs, nasal epithelium, brain, and skin. But, the maximum drug metabolism takes place in the liver. ${ }^{1}$ The in vivo metabolism of drugs has been categorized into two groups namely, phase I and phase II metabolism reactions. Hydrophilicity of drugs increases, as an outcome of drug metabolism so that it can easily be excreted as a fate of foreign material entered into the body and toxicity (if any) abates. ${ }^{2}$
Submission Date: 01-06-2021; Revision Date: 27-08-2021; Accepted Date: 09-11-2021.

DOI: 10.5530/ijper.56.1s.43 Correspondence: Mr. Lovekesh Mehta Research Scholar, Amity Institute of Pharmacy (AIP), Amity University, Noida-201301, Uttar Pradesh, INDIA E-mail: mehta.lovekesh@ gmail.com

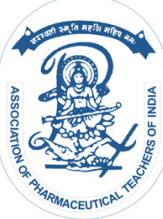

www.ijper.org 
Belinostat, a histone deacetylase (HDAC) inhibitor is one among the first four FDA-approved drugs used to treat relapsed or refractory peripheral T-cell lymphoma. ${ }^{3-6} \mathrm{In}$ addition, belinostat is also an established drug to enhance the immune response required in lung adenocarcinoma 7,8 and melanoma 9 via PD-1 (Programmed Death1) activation either alone or in combination. ${ }^{10,11}$ The structure of the belinostat is shown in Figure 1.

Wide range of, in vitro methods have been developed in which the metabolism of drugs is studied outside the body using rodent's liver extract itself or its microsomal fraction or isolated liver enzymes. The metabolites then formed are identified and characterized. The analysis of metabolites formed is a very challenging task, therefore, with the advancement in technology, new analytical methods are available for the qualification and quantification which are then used to identify novel metabolites. Liquid chromatography coupled with mass spectrometry (LC-MS) acts as the most powerful analytical tool for the identification of drug metabolites. In literature, there are several LC-MS/MS and HPLC methods available for analysis of degradation products of anticancer drugs. ${ }^{12-14}$

The present work was designed to study in vitro metabolism of anticancer drug, belinostat using rat liver microsomes (RLM) and to characterize the formed metabolites using LC-MS/MS. The study also established the precise and rapid HPLC and LC-MS/ MS method to separate, identify and characterize the formed Phase-I metabolites of belinostat. Based on the data of the sophisticated LC-MS/MS technique, the probable structures of both metabolites of belinostat were proposed which will assist the researchers working in the niche area of drug development. Moreover, kinetics of belinostat in presence of enzymes set present in the RLM was also established in this study.

\section{MATERIALS AND METHODS}

\section{Chemicals and Reagents}

Pharmaceutical grade belinostat standard was procured from MSN Laboratories Limited, Hyderabad, as a

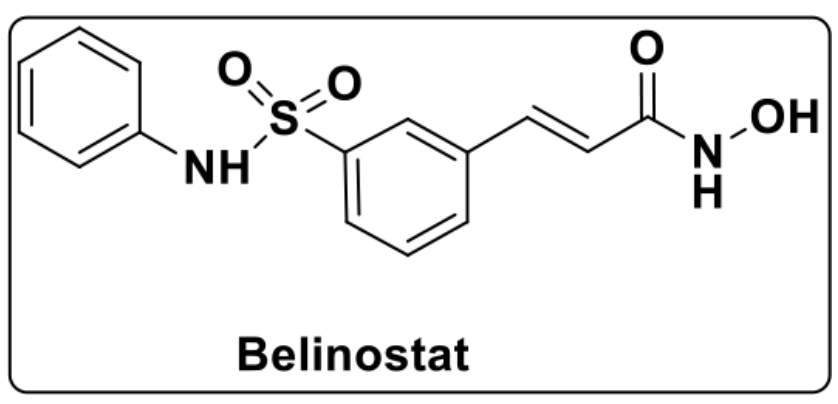

Figure 1: Chemical structure of Belinostat. gift sample with a purity of more than $99.7 \% \mathrm{w} / \mathrm{w}$. All solvents, chemicals and reagents used in the current study were of analytical grade procured from Merck life sciences, Bangalore, India. LC-MS grade acetonitrile $(\mathrm{ACN})$, formic acid was procured from JT Baker. Tris buffer, $\mathrm{NaOH}$ was purchased from Merck (Mumbai, India). The solutions used in the study were filtered through $0.22 \mu \mathrm{m}$ filter paper (Millipore, India). Purification of water used for the preparation of solution was done by the Milli-Q-Plus system.

\section{Preparation of Rat Liver Microsomes}

Male Wistar rat used for liver procurement was approved for experimentation from ethical committee (IAEC/56/1060) and was not exposed to any form of medication before. The entire procedure was performed at refrigerated $\left(0-4^{\circ} \mathrm{C}\right)$ temperature using an ice bath. The excised livers ( $20 \mathrm{~g}$ per batch and 5 such batches) were frozen, finely cut with scissors and the excised liver was homogenized using four times $(40 \mathrm{~mL})$ the weight of the liver of tris buffer ( $\mathrm{pH}$ 7.4. The homogenate was subjected to centrifugation at $4{ }^{\circ} \mathrm{C}$ for about 10 min the centrifugation chamber and the supernatant was maintained at $4^{\circ} \mathrm{C}$ and solid calcium chloride was added to the supernatant (10 $\mathrm{mM}$ final concentration) with continuous stirring. The firmly packed pellets with the help of a homogenizer obtained and store in cold condition $\left(-70^{\circ} \mathrm{C}\right)$ for further use.

\section{Microsomal Metabolism of Belinostat}

Microsomal drug metabolism was carried out by incubating microsomes of rat liver (RLM) with belinostat in EP tubes having a capacity of $1.5 \mathrm{~mL}$. Incubation system comprised of Tris buffer (0.05 M, pH 7.4), RLM, NADPH generating system and belinostat solution (prepared with the final concentration of $0.2 \mathrm{mM}$ ) with a total volume of $1.0 \mathrm{~mL}$. NADPH generated solution comprises glucose 6-phosphate, NADP and magnesium chloride. Incubation was performed for $30 \mathrm{~min}$ at about $37^{\circ} \mathrm{C}$ and the metabolic reaction was enhanced with the help of the NADPH generating system. ${ }^{15}$ After 30 min of incubation, the metabolic reaction was quenched by $1.0 \mathrm{~mL} 0.5 \mathrm{M} \mathrm{NaOH}$. Control incubation was also carried out simultaneously without the addition of rat liver microsomes in the incubation system to define the contamination or interference obtained due to incubation components. Before injecting into the UPLC system, samples were kept in refrigerated conditions. The incubation mixture was filtered through a $0.22 \mu \mathrm{m}$ filter before injecting into the UPLC system. All the reactions were performed in triplicate. 


\section{Sample preparation for HPLC analysis}

Samples were extracted using ethyl acetate before injecting them into the HPLC system. After extraction, both the aqueous and organic layers were allowed to separate. The organic layer (Ethyl acetate) was evaporated to at $45^{\circ} \mathrm{C}$ until dry on a water bath for nearly about $2-3$ h. After drying, the volume was made up with diluent to achieve the final nominal concentration and injected into the UPLC system.

\section{Optimized HPLC Conditions}

The analysis of the drug was executed on an ultraperformance liquid chromatographic (UPLC) system equipped with a Quaternary Solvent Manager (QSM), a $20 \mu \mathrm{L}$ injection loop (Rheodyne 7725i) and a PDA detector. The chromatograms were analyzed using Empower 2.0 software. Belinostat and its metabolites were separated with a reverse-phase BEH C18 column $(1000 \mathrm{~mm} \times 2.1 \mathrm{~mm} \times 1.7 \mu \mathrm{m})$. The composition of the mobile phase was formic acid and acetonitrile with elution in gradient mode. The analysis was performed at a flow rate of $0.3 \mathrm{~mL} / \mathrm{min}$. Run time was kept at $25 \mathrm{~min}$ and absorbance was monitored at $210 \mathrm{~nm}$. Metabolites were recognized by evaluation of Retention times (RT) and co-injection of standards (spiking the metabolite mixture with the authentic standard of belinostat). Optimized chromatographic conditions and gradient programming were given in Table 1 and Table 2 respectively.

\section{LC-MS Instrumentation}

A new method for the identification and characterization of in vitro metabolites by LC-MS technology was developed. Chromatographic separation was carried

\begin{tabular}{|c|c|}
$\begin{array}{c}\text { Table 1: Chromatographic conditions for optimized } \\
\text { LCMS method. }\end{array}$ \\
\hline Parameter & Condition \\
\hline Column & $\begin{array}{c}\text { ACQUITY UPLC BEH C- } \\
(100 \mathrm{~mm} \times 2.1 \mathrm{~mm}, 1.7 \mu \mathrm{m})\end{array}$ \\
\hline Eluent A & $0.1 \%$ Formic acid in water \\
\hline Eluent B & Acetonitrile \\
\hline Detector, Wavelength & PDA, $210 \mathrm{~nm}$ \\
\hline Injection volume & $5 \mu \mathrm{L}$ \\
\hline Flow Rate & $0.3 \mathrm{~mL} \mathrm{~min}{ }^{-1}$ \\
\hline Diluent & Water: Acetonitrile $(50: 50) \mathrm{v} / \mathrm{v}$ \\
\hline Column Temperature & $45^{\circ} \mathrm{C}$ \\
\hline Auto sampler Temperature & $10^{\circ} \mathrm{C}$ \\
\hline Run time & $25 \mathrm{~min}^{-1}$ \\
\hline Nominal concentration & $100 \mu \mathrm{g} \mathrm{mL}{ }^{-1}$ \\
\hline
\end{tabular}

PDA is photodiode array detector
Table 2: Gradient program used for analysis.

\begin{tabular}{|c|c|c|}
\hline Time (minutes) & Eluent A (\%) & Eluent B (\%) \\
\hline 0.01 & 70 & 30 \\
\hline 10 & 50 & 50 \\
\hline 15 & 30 & 70 \\
\hline 24.9 & 30 & 70 \\
\hline 25 & 70 & 30 \\
\hline
\end{tabular}

out on an Agilent Technologies 1200 series LC system equipped with a column Waters BEH C18 column (2.1 $\mathrm{mm} \mathrm{id} \times 100 \mathrm{~mm}, 1.7 \mu$ ). A mobile phase consisting of A: $0.05 \%$ Formic acid in water and B: Acetonitrile used at a gradient condition. The flow rate was maintained at $0.3 \mathrm{~mL} \mathrm{~min}^{-1}$. The mobile phase was degassed on the sonicator and filtered through a $0.22 \mu \mathrm{m}$ before the start of the analysis. The sample injection volume was $5 \mu \mathrm{L}$. LC system coupled with an MS of Agilent technology with triple Quadrupole mass analyzer 6460, operated using ESI (electrospray ionization) source in negative mode. Analysis was performed in selected ion monitoring (SIM) mode. It was chosen for the identification of the interested compound. The following parameters were set: capillary voltage: $3500 \mathrm{~V}$, nebulizer voltage: $500 \mathrm{~V}$, nebulizer pressure: 35 psi, sheath gas flow: $10 \mathrm{~L} / \mathrm{min}$, Sheath gas temperature: $350^{\circ} \mathrm{C}$, gas temperature: $300^{\circ} \mathrm{C}$ with a gas flow: $10 \mathrm{~L} / \mathrm{min}$. Mass hunter software was used to do the acquisition and processing in the LC-MS system.

\section{Enzymes Kinetic Evaluation}

Before evaluation of the enzyme kinetics, the environments for incubation period and concentrations of proteins in rat liver microsomes were established. Protein concentration in rat liver microsomes was found to be $200 \mathrm{mg} \mathrm{mL}^{-1}$. The rate of formation of M1 and M2 were linear within $30 \mathrm{~min}$ at $0.5 \mathrm{mg} \mathrm{mL}^{-1}$ of RLM. Therefore, for enzyme kinetic studies, the incubation mixture contained Tris- $\mathrm{HCl}$ buffer (100 mM, pH 7.4), NADPH $(1 \mathrm{mM})$, rat liver microsomes $\left(0.5 \mathrm{mg} \mathrm{mL}^{-1}\right)$ a series concentration of belinostat. Ten different concentration of substrate $(20.0,40.0,60.0,80.0,100.0$, $120.0,140.0,160.0,180.0$ and $200.0 \mathrm{mM}$ ) were assessed in triplicate for enzyme kinetics evaluation. The incubation time was $30 \mathrm{~min}$ and the temperature condition was set at $37^{\circ} \mathrm{C}$.

\section{RESULTS AND DISCUSSION}

\section{HPLC Method Evaluation}

For UPLC method development and standardization assay, the samples were prepared as per the specified 
incubation conditions. Standard belinostat eluted at 8.3 min under the stated developed UPLC conditions. When RLM incubated belinostat solutions were spiked, two metabolites were observed based on their retention time (RTs). The unchanged belinostat was eluted at 8.3 min. The metabolites M1 and M2 were eluted at 2.4 and 7.6 min respectively. PDA analysis showed value of peak angle less than the peak threshold representing all peaks are pure and ruled out possibility of co-elution any other peak or metabolite in RLM treated belinostat solution. The chromatogram representing control sample (without RLM treatment) and microsomal incubation sample are shown in supplementary Figure S1-S2. The UV spectrum scan chromatogram of belinostat and both Phase-I metabolites are given in supplementary Figure S3-S5.

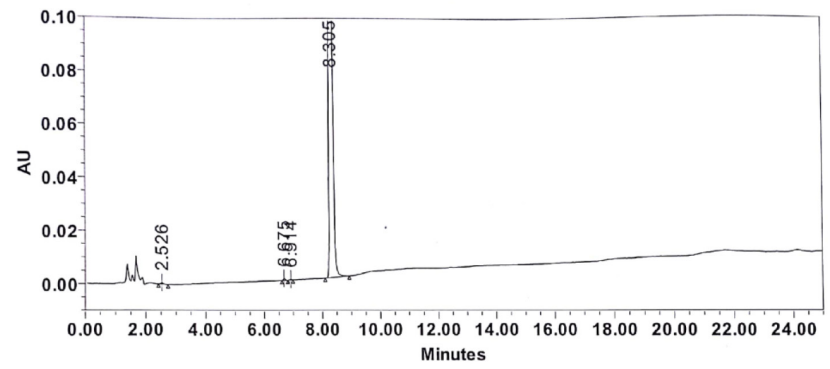

Supplementary Figure 1: Chromatogram representing belinostat without microsomal incubation (control).

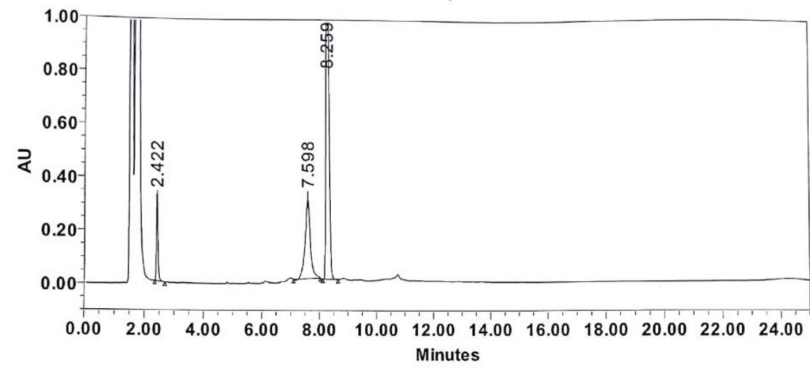

Supplementary Figure 2: Chromatogram representing microsomal incubation of belinostat.

Match Plot

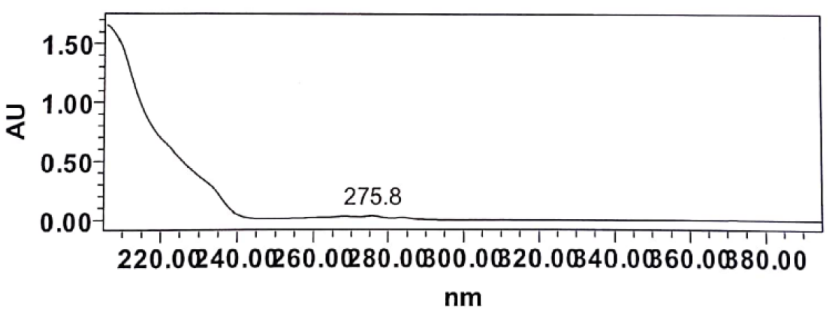

Supplementary Figure 3: Chromatogram showing UV spectra of Belinostat.

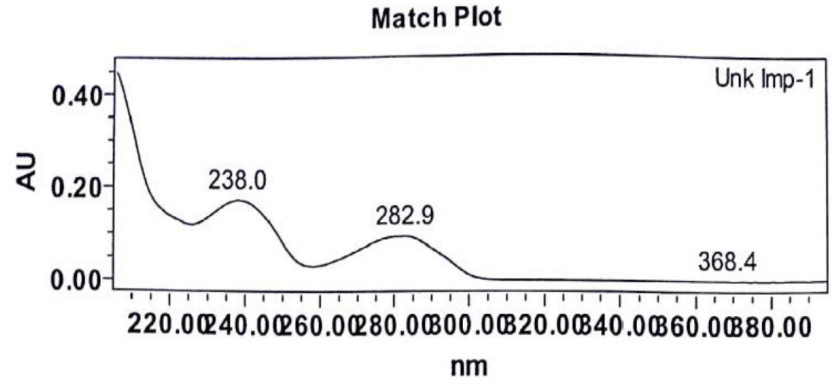

Supplementary Figure 4: Chromatogram showing UV spectra of Metabolite M1.

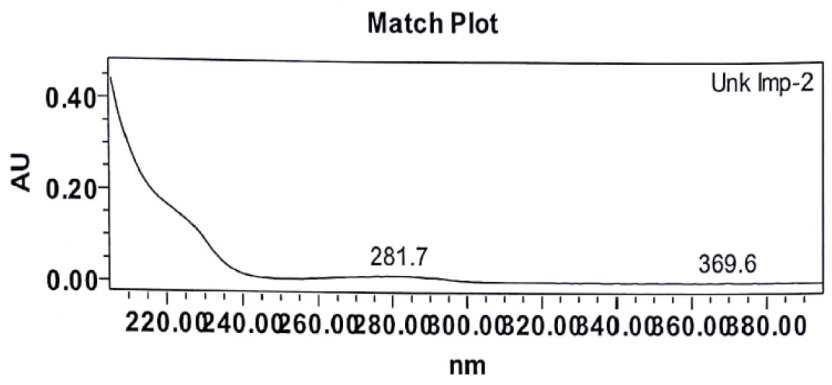

Supplementary Figure 5: Chromatogram showing UV spectra of Metabolite M2.

\section{Metabolic Fate of Belinostat}

The extracted sample was further analyzed for metabolite identification by the LC-MS technique. A total of two Phase-I metabolites were observed using rat liver microsomes. Total ions chromatograms of the test incubation showed three peaks for M1, M2 and belinostat respectively. The belinostat has an $\mathrm{m} / \mathrm{z}$ value of $317.0670 \mathrm{Da}[\mathrm{M}-\mathrm{H}]^{-}$in standard and incubation samples under the same LC/MS conditions. Metabolite M1 was obtained with a molecular weight of $\mathrm{m} / \mathrm{z}$ at $301.0719 \mathrm{Da}[\mathrm{M}-\mathrm{H}]$; , and the molecular formula was calculated as $\mathrm{C}_{15} \mathrm{H}_{13} \mathrm{~N}_{2} \mathrm{O}_{3} \mathrm{~S}$, which was 16 amu lesser than $[\mathrm{M}-\mathrm{H}]^{-}$of belinostat, indicating that dehydroxylation reaction resulted in the formation of belinostat amide. The proposed fragmentation pathway and MS/MS bar graph of M1 is shown in Figure 3. Metabolite M1 was eluted at RT 2.3 min indicating that the formed metabolite is more polar than belinostat. MS/MS spectra of M1 results in formation of 258.0637 $\mathrm{Da} \quad\left(\mathrm{C}_{14} \mathrm{H}_{12} \mathrm{NO}_{2} \mathrm{~S}\right), \quad 237.1086 \mathrm{Da} \quad\left(\mathrm{C}_{12} \mathrm{H}_{17} \mathrm{~N}_{2} \mathrm{OS}^{-}\right)$, 194.1019 ( $\left.\mathrm{C}_{10} \mathrm{H}_{12} \mathrm{NOS}^{-}\right)$, 92.0530Da $\left(\mathrm{C}_{6} \mathrm{H}_{6} \mathrm{~N}^{-}\right)$. Based on the above-supporting data, Metabolite M1 was found to be 3-(3-(N-phenylsulfamoyl) phenyl) acrylamide.

Metabolite M2 was obtained with a molecular weight of $\mathrm{m} / \mathrm{z}$ at $302.0557 \mathrm{Da}[\mathrm{M}-\mathrm{H}]-$, and the molecular formula was calculated as $\mathrm{C}_{15} \mathrm{H}_{12} \mathrm{NO}_{4} \mathrm{~S}$, which was $15 \mathrm{amu}$ lesser than $[\mathrm{M}-\mathrm{H}]$ - of belinostat, indicating deamination of N-Hydroxyformamide and further carboxylation 


\begin{tabular}{|c|c|c|c|c|c|c|}
\hline \multicolumn{6}{|c|}{ Table 3: Retention time, MS/MS and characterization of belinostat and its metabolites. } \\
\hline Compound & $\begin{array}{c}\text { Retention } \\
\text { time (min) }\end{array}$ & $\begin{array}{c}{[\mathrm{M}-\mathrm{H}]} \\
(\mathrm{m} / \mathbf{z})\end{array}$ & $\begin{array}{c}\text { Folecular } \\
\text { weight }\end{array}$ & $\begin{array}{c}\text { Identification } \\
\text { Metabolic } \\
\text { pathways }\end{array}$ \\
\hline M0 & 8.26 & 317.0670 & $\begin{array}{c}299.0548,283.0605,207.0969, \\
181.0233,165.0042,154.0125, \\
144.0463,92.0526\end{array}$ & 318.0674 & Belinostat & Parent \\
\hline M1 & 2.42 & 301.0719 & $258.0637,237.1086,194.1019$, & 302.0725 & $\begin{array}{c}\text { Belinostat } \\
\text { amide }\end{array}$ & Reduction \\
\hline M2 & 7.60 & 302.0557 & $\begin{array}{c}260.0443,258.0646155 .0080, \\
119.0533,92.0529\end{array}$ & 303.0565 & $\begin{array}{c}\text { Belinostat acid } \\
\text { Deamination }\end{array}$ \\
\hline
\end{tabular}

Mo: Belinostat
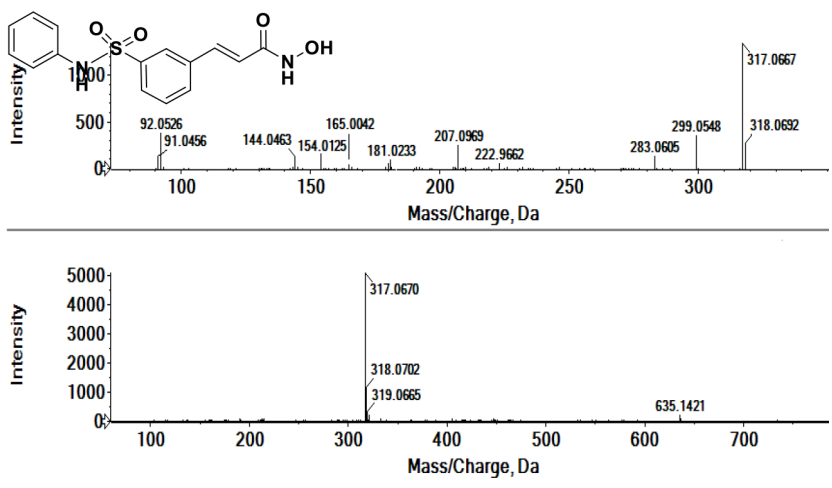

Figure 2: Proposed fragmentation pathway and MSMS bar graph of Belinostat.
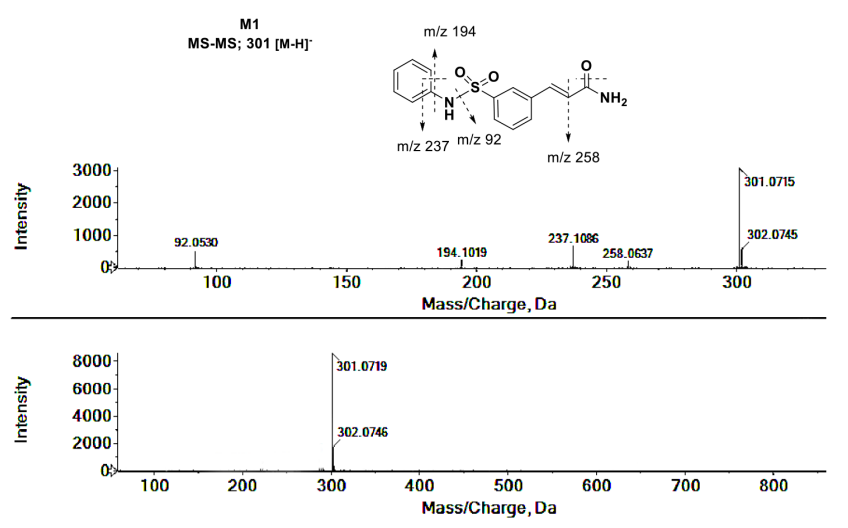

Figure 3: Proposed fragmentation pathway and MSMS bar graph of metabolite M2.

resulted in the formation of belinostat acid. The proposed fragmentation pathway and MS/MS bar graph of M2 is shown in Figure 4. Metabolite M2 was eluted at RT $7.5 \mathrm{~min}$ indicating that the formed metabolite is more polar than belinostat. MS/MS spectra of M2 results in the formation of $260.0443 \mathrm{Da}\left(\mathrm{C}_{14} \mathrm{H}_{14} \mathrm{NO}_{2} \mathrm{~S}\right)$, $258.0646 \mathrm{Da}\left(\mathrm{C}_{14} \mathrm{H}_{12} \mathrm{NO}_{2} \mathrm{~S}\right), 155.0080 \mathrm{Da}\left(\mathrm{C}_{7} \mathrm{H}_{7} \mathrm{O}_{2} \mathrm{~S}\right)$, $119.0533 \mathrm{Da}\left(\mathrm{C}_{8} \mathrm{H}_{7} \mathrm{O}^{-}\right), 92.0529 \mathrm{Da}\left(\mathrm{C}_{6} \mathrm{H}_{6} \mathrm{~N}^{-}\right)$. Based on the above-supporting data, Metabolite M2 was found to be 3-(3-(N-phenylsulfamoyl)phenyl)acrylic acid.
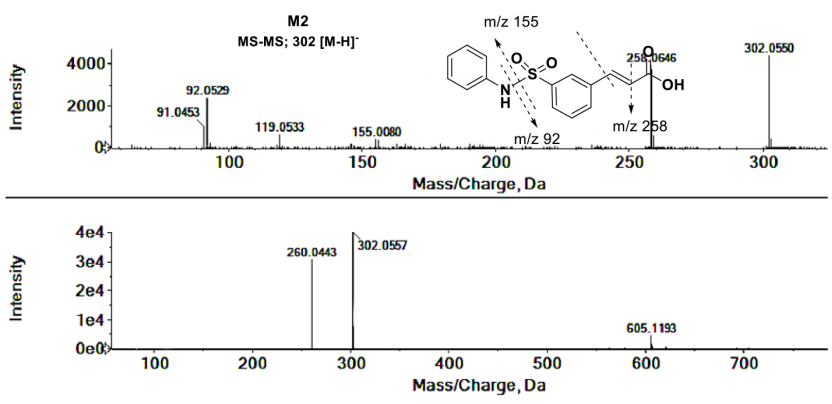

Figure 4: Proposed fragmentation pathway and MSMS bar graph of metabolite M2.

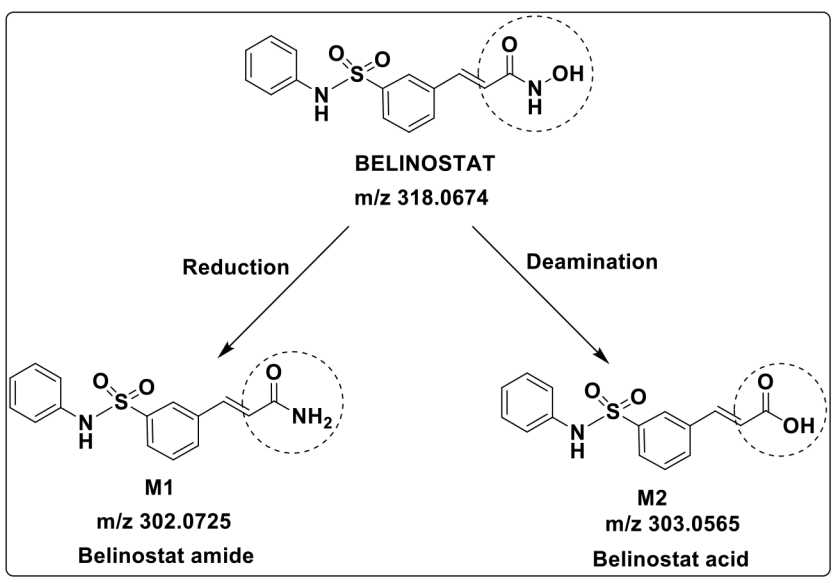

Figure 5: Proposed metabolic pathway of belinostat in rat liver microsomes (in vitro).

The metabolite M2 accounting for the largest percent (25.71\%) whereas metabolite M1 was a minor metabolite, accounting for a lesser percent (7.42\%). The retention times, measured and theoretical masses, mass errors, and characteristic fragment ions of the proposed metabolites are summarized in Table 3. The structures of metabolites were characterized based on their accurate masses, fragment ions, and retention times. The proposed fragmentation pathway and MS/ MS bar graph of belinostat is shown in Figure 2 and the 
proposed metabolic pathway of belinostat in rat liver microsomes (in vitro) is shown in Figure 5.

In vivo metabolism is spontaneously amenable to phase II reaction along with phase I because precursor to be utilized in the anabolic reaction are available. But studies presented the feasibility of type II reaction by adding suitable precursor (e.g. uridine diphosphate-glucuronic acid, 3'-phosphoadenosine-5'-phosphosulfate) externally along with liver preparation containing suitable enzyme $/ \mathrm{s}^{16}$. In this study, sulfation was also attempted by the use of RLM with sodium sulfate as a possible phase II metabolism substrate instead of promising 3'-phosphoadenosine-5'-phosphosulfate (PAPS). ${ }^{17}$ The metabolite outcome with sodium sulfate was not observed in our experimental conditions or protocols, which may be attributed to absence of

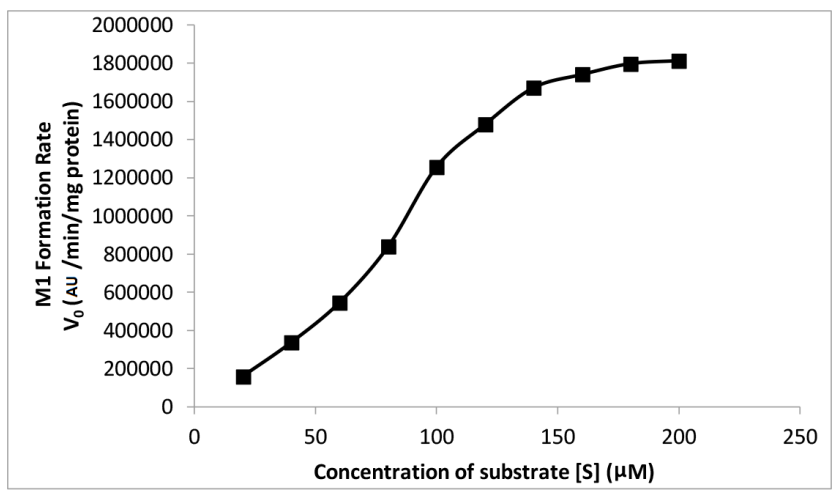

Figure 6: Michaelis-Menten plot for M1 formation from belinostat metabolism by RLMs. Each point on the curve represents the mean \pm standard deviation of triplicate determination.

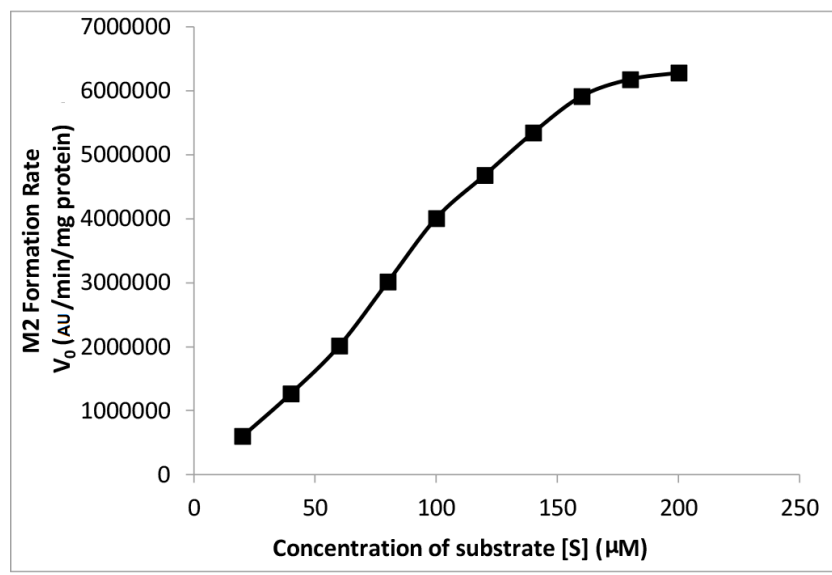

Figure 7: Michaelis-Menten plot for M2 formation from belinostat metabolism by RLMs. Each point on the curve represents the mean \pm standard deviation of triplicate determination. sulfotransferase enzyme in the RLM or if present not showing affinity for sodium sulfate. In another study of belinostat, in vivo metabolic degradation in human plasma also showed the formation of five metabolites but in present experimental setup using rat liver microsomes (RLM), two among five were observed. Such difference in results is attributable to the utilization of specific organ extract part i.e. RLM instead of intact human physiological system to treat the drug. ${ }^{18}$

Kinetic Study of Belinostat in Rat Liver Microsomes

The rate of formation of metabolites M1 and M2 from belinostat by using rat liver microsomes well suited to the Michaelis-Menten kinetic equation. The MichaelisMenten enzyme kinetic plots of Metabolite M1 and M2 are given in Figure 6 and Figure 7 respectively. The straight line obtained from the double reciprocal plot

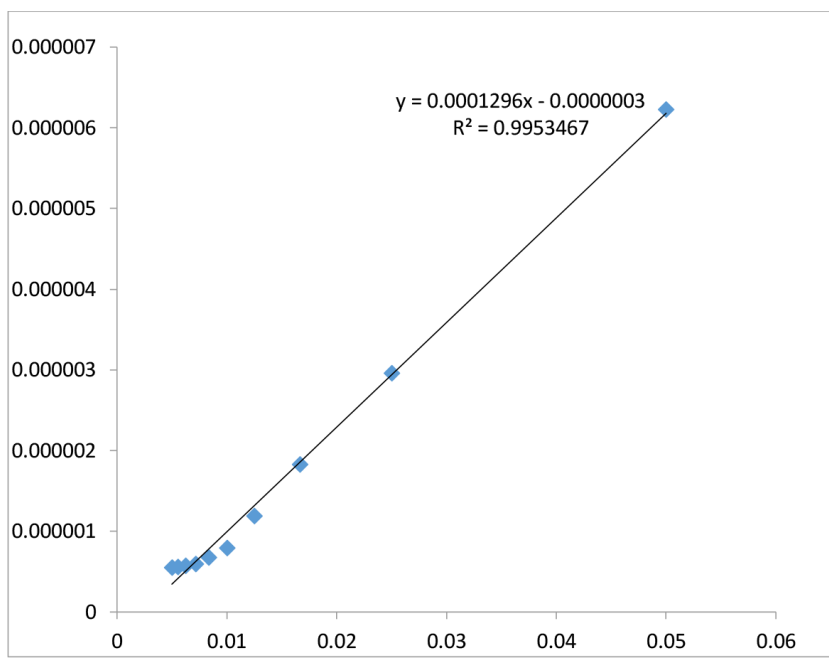

Supplementary Figure 6: Straight line curve for Metabolite M2.

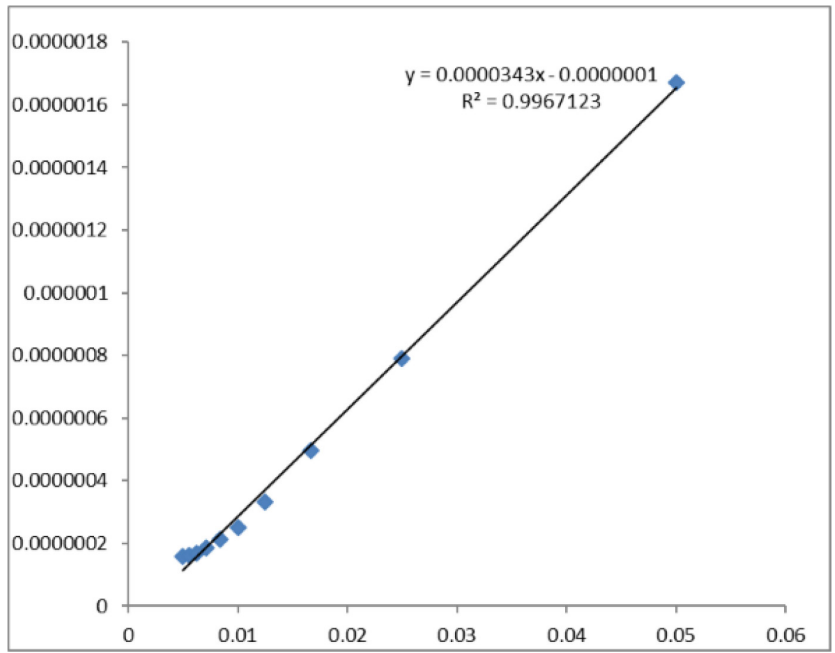

Supplementary Figure 7: Straight line curve for Metabolite M2. 
(1/S vs I/V) provided a slope that corresponds to $\mathrm{K}_{\mathrm{m}}$ / $\mathrm{V}_{\max }$ and intercept $\left(1 / \mathrm{V}_{\max }\right)$ for both metabolites. The regression equation was $\mathrm{y}=0.0001296 \mathrm{x}-0.0000003$ and the correlation coefficient (r2) was 0.9953 for M1 and regression equation was $\mathrm{y}=0.0000343 \mathrm{x}-0.0000001$ and the correlation coefficient (r2) was 0.9967 for M2 metabolite given in supplementary Figure-S6-S7. With the help of a double reciprocal plot, we obtained the $\mathrm{K}_{\mathrm{m}}$ and $\mathrm{V}_{\max }$ values of both the metabolites. The $K_{\mathrm{m}}$ values for the formation of M1 and M2 were $432 \pm 18.16$ and $343 \pm 14.34 \mu \mathrm{M}$ in rat liver microsomes, respectively. $\mathrm{K}_{\mathrm{m}}$ is a characteristic of any enzyme which show affinity of an enzyme towards its range of substrates or range of enzymes for particular substrate. At constant enzyme concentration, lower enzyme $\mathrm{K}_{\mathrm{m}}$ value towards particular substrate suggests more affinity ${ }^{19-20}$. Since the RLM is not a pure enzyme, neither the content of different enzyme is known so affinity $/ \mathrm{K}_{\mathrm{m}}$ value will also depend upon the content of enzyme along with the enzyme affinity. Hence, $\mathrm{K}_{\mathrm{m}}$ value obtained in this study was less in case of M2 so it is conclusive that the enzyme set present in the RLM has more affinity and/or content of converting substrate belinostat into corresponding acid in comparison to enzyme set for converting belinostat to corresponding amide.

\section{CONCLUSION}

In summary, our present study focused on prediction of in-vitro drug metabolism of newly approved FDA drug, belinostat. The study also includes determination of metabolic pathways of the formed metabolites which are needed to clarify the effects of drug-metabolizing enzymes in-vitro which will further assist in expanding the knowledge about hepatic biotransformation in-vivo. Further, enzyme kinetic parameters $\left(K_{\mathrm{m}}\right.$ and $\left.\mathrm{V}_{\max }\right)$ were also evaluated that will assist in pharmacokinetic studies of drug. Two metabolites of belinostat were identified and characterized using LC-MS/MS. Metabolism of belinostat was found to generate reductive as well as a deaminated metabolite. The method employed for separation and detection is quite accurate and able to detect the metabolites. The formed phase I metabolites might represent an important step in designing and planning future studies with belinostat.

\section{ACKNOWLEDGMENT}

I would like to thank the KIET Group of Institutions and Department of Pharmaceutical Science, Amity University, Noida for encouragement and providing all the necessary research facilities to carry out RP-UPLC and LC-MS/MS related work. I am also very much thankful to the MSN laboratory, Hyderabad for providing pure belinostat drug as a gift sample.

\section{CONFLICT OF INTEREST}

The authors declare that there are no conflicts of interest.

\section{ABBREVIATIONS}

${ }^{\circ} \mathrm{C}$ : Degree Celsius; ACN: Acetonitrile; AU: Arbitrary Unit; BEH: Ethylene Bridged Hybrid; BLN: Belinostat; CPCSEA: The Committee for the Purpose of Control and Supervision of Experiments on Animals; Da: Dalton; EP: Eppendorf; FDA: Food and Drug Administration; g: Gram; HDAC: Histone deacetylase; HPLC: High Performance Liquid Chromatography; hr: hour $\mathbf{K}_{\mathrm{m}}$ : Michaelis constant; $\mathbf{L C}$ : Liquid chromatography; min: Minute; mL: Milliliter; MS: Mass Spectrometry; NADP: Nicotinamide adenine dinucleotide phosphate; NADPH: Nicotinamide Adenine Dinucleotide Phosphate; NaOH: Sodium hydroxide; PAPS: 3'-phosphoadenosine-5'phosphosulfate; PD-1: Programmed Death-1; PDA: Photo diode array; QSM: Quaternary Solvent Manager; RLM: Rat liver microsomes; RP: Reverse-Phase; RT: Retention time; UPLC: Ultra Performance Liquid Chromatography; UV: Ultra Violet; $\mathbf{V}_{\text {max }}$ : Maximum rate of reaction

\section{REFERENCES}

1. Schonborn JL. The role of the liver in drug metabolism anaesthesia tutorial of the week 179.

2. Prakash C, Shaffer CL, Nedderman A. Analytical strategies for identifying drug metabolites. Mass spectrometry reviews. 2007;26(3):340-69.

3. Poole RM. Belinostat: first global approval. Drugs. 2014 Sep;74(13):1543-54.

4. Zheng H, Zhao W, Yan C, Watson CC, Massengill M, Xie M, Massengill C, Noyes DR, Martinez GV, Afzal R, Chen Z. HDAC inhibitors enhance T-cell chemokine expression and augment response to PD-1 immunotherapy in lung adenocarcinoma. Clinical Cancer Research. 2016;22(16):4119-32.

5. Woods DM, Sodré AL, Villagra A, Sarnaik A, Sotomayor EM, Weber J. HDAC inhibition upregulates PD-1 ligands in melanoma and augments immunotherapy with PD-1 blockade. Cancer immunology research. 2015;3(12):1375-85.

6. Banik D, Moufarrij S, Villagra A. Immunoepigenetics combination therapies: an overview of the role of HDACs in cancer immunotherapy. International journal of molecular sciences. 2019;20(9):2241.

7. Bai Y, Ahmad D, Wang T, Cui G, Li W. Research advances in the use of histone deacetylase inhibitors for epigenetic targeting of cancer. Current topics in medicinal chemistry. 2019;19(12):995-1004.

8. Mottamal M, Zheng S, Huang TL, Wang G. Histone deacetylase inhibitors in clinical studies as templates for new anticancer agents. Molecules. 2015 Mar;20(3):3898-941.

9. Ziemka-Nalecz M, Jaworska J, Sypecka J, Zalewska T. Histone deacetylase inhibitors: a therapeutic key in neurological disorders?. Journal of Neuropathology \& Experimental Neurology. 2018;77(10):855-70.

10. Halili MA, Andrews MR, Sweet MJ, Fairlie DP. Histone deacetylase inhibitors in inflammatory disease. Current topics in medicinal chemistry. 2009 Feb $1 ; 9(3): 309-19$. 
11. Yoon S, Eom GH. HDAC and HDAC inhibitor: from cancer to cardiovascular diseases. Chonnam medical journal. 2016;52(1):1-1.

12. Mehta L, Naved T, Grover P, Bhardwaj M, Mukherjee D. LC and LC-MS/ MS studies for identification and characterization of new degradation products of ibrutinib and elucidation of their degradation pathway. Journal of Pharmaceutical and Biomedical Analysis. 2021;194:113768.

13. Mehta L, Naved T, Grover P, Bhardwaj M, Mukherjee D. Development and Validation of Novel and Highly Sensitive Stability-Indicating Reverse Phase Ultra Performance Liquid Chromatography Method for Quantification of Ibrutinib and its ten Degradation Products. Indian Journal of Pharmaceutical Sciences. 2020;82(6):958-66.

14. Mehta L, Naved T, Grover P, Bhardwaj M, Mukherjee D, Vennapu DR. Identification and characterization of new degradation products of belinostat using UHPLC-Q-TOF-MS/MS and in silico toxicity prediction. Journal of Liquid Chromatography \& Related Technologies. 2021 Apr 26:1-3.

15. Jia L, Liu X. The conduct of drug metabolism studies considered good practice (II): in vitro experiments. Current drug metabolism. 2007;8(8):822-9.

16. Zhang C, Guo S, Zhong Q, Zhang Q, Hossain A, Zheng S, Wang G. Metabolism and pharmacokinetic study of the boron-containing prodrug of belinostat (ZL277), a pan HDAC inhibitor with enhanced bioavailability. Pharmaceuticals. 2019;12(4):180

17. Weththasinghe SA, Waller CC, Fam HL, Stevenson BJ, Cawley AT, McLeod MD. Replacing PAPS: In vitro phase II sulfation of steroids with the liver S9 fraction employing ATP and sodium sulfate. Drug testing and analysis. 2018;10(2):330-9.

18. Kiesel BF, Parise RA, Tjørnelund J, Christensen MK, Loza E, Tawbi H, Chu E, Kummar S, Beumer JH. LC-MS/MS assay for the quantitation of the HDAC inhibitor belinostat and five major metabolites in human plasma. Journal of pharmaceutical and biomedical analysis. 2013;81:89-98.

19. Kumar S, Jana AK, Dhamija I, Singla Y, Maiti M. Preparation, characterization and targeted delivery of serratiopeptidase immobilized on aminofunctionalized magnetic nanoparticles. European Journal of Pharmaceutics and Biopharmaceutics. 2013;85(3):413-26.

20. Kumar S, Jana AK, Dhamija I, Maiti M. Chitosan-assisted immobilization of serratiopeptidase on magnetic nanoparticles, characterization and its target delivery. Journal of drug targeting. 2014;22(2):123-37.

\section{SUMMARY}

- Biotransformation study of BLN was examined using isolated microsomes from rat liver. Incubation of microsomes was carried out in phosphate buffer $\mathrm{pH} 7.4$ by using micro centrifuge tube. Extraction of the incubated microsomes was carried out in ethyl acetate. All the extracted samples were analyzed first by RP-UPLC technique and newly formed metabolites were identified and characterized with the help of ultrahigh performance liquid chromatography which is interlinked with tandem quadrupole time-of-flight mass spectrometry.

- The resultant chromatographs were compared with the authentic standard. Different elution times were recorded in RP-UPLC method. The unchanged belinostat was eluted at $8.3 \mathrm{~min}$. The metabolites M1 and M2 were eluted at 2.4 and 7.6 min respectively. Out of the three peaks, two metabolites of BLN were detected in LC-MS study in the rat liver microsomal incubation in vitro.

- Based on LC-MS/MS data, metabolite M1 identified as belinostat amide (reductive metabolite) and metabolite M2 identified as belinostat acid (deaminated belinostat). The metabolite M2 accounting for the largest percent (25.71\%) whereas metabolite M1 was a minor metabolite, accounting for a lesser percent $(7.42 \%)$. In addition, enzyme kinetic parameters $\left(\mathrm{K}_{\mathrm{m}}\right.$ and $\left.\mathrm{V}_{\max }\right)$ were also evaluated that will assist in pharmacokinetic studies of drug. The rate of formation of metabolites M1 and M2 from belinostat by using rat liver microsomes well suited to the Michaelis-Menten kinetic equation. $\mathrm{K}_{\mathrm{m}}$ value obtained in this study was less in case of M2 so it is conclusive that the enzyme set present in the RLM has more affinity and/or content of converting substrate belinostat into corresponding acid in comparison to enzyme set for converting belinostat to corresponding amide.

\section{PICTORIAL ABSTRACT}

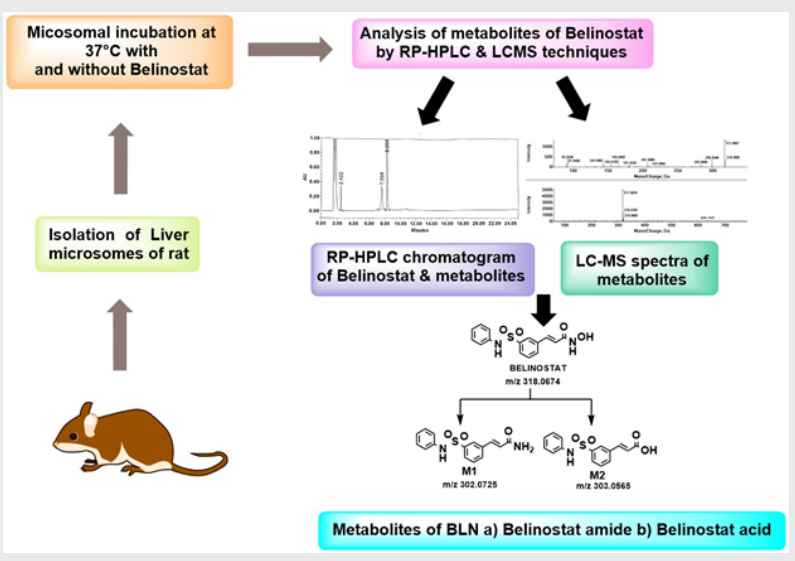




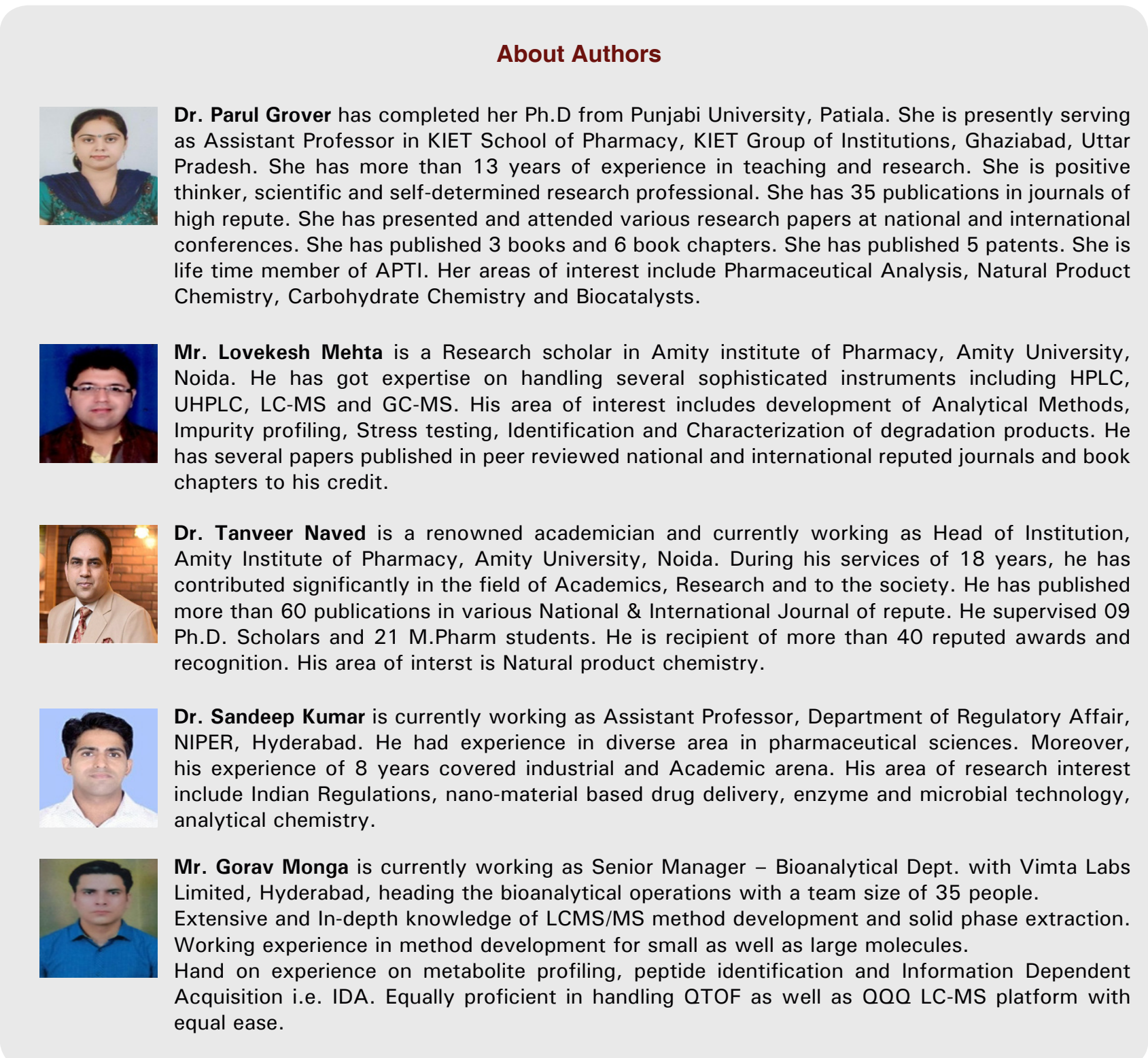

Cite this article: Grover P, Mehta L, Naved T, Kumar S, Monga G. Identification and Characterization of in vitro Metabolites of Belinostat by Rat Liver Microsomes using Ultra Performance Liquid Chromatography Coupled with Tandem Mass Spectrometry. Indian J of Pharmaceutical Education and Research. 2022;56(1s):s58-s66. 